\title{
Factors Associated with Infection of the Central Venous Catheter for Hemodialysis in Ouagadougou (Burkina Faso)
}

\author{
Gérard Coulibaly ${ }^{*}$, Gildas Ilboudo ${ }^{1}$, Kongnimissom Apoline Sondo², \\ Adama Roger Karambiri' ${ }^{1}$, François P. Kissou ${ }^{1}$, Adama Lengani1 \\ ${ }^{1}$ Department of Nephrology and Hemodialysis, Yalgado Ouedraogo University Hospital Center (YO-UHC), \\ Ouagadougou, Burkina Faso \\ ${ }^{2}$ Department of Infectious Diseases, Yalgado Ouedraogo University Hospital Center (YO-UHC), Ouagadougou, \\ Burkina Faso \\ Email: "coulibalygerard@hotmail.fr
}

Received 2 December 2015; accepted 27 December 2015; published 30 December 2015

Copyright (C) 2015 by authors and Scientific Research Publishing Inc.

This work is licensed under the Creative Commons Attribution International License (CC BY). http://creativecommons.org/licenses/by/4.0/

(c) (i) Open Access

\begin{abstract}
Goal: The goal is to evaluate infections associated with central venous catheters of hemodialysis (CRI) in hemodialysis patients in Ouagadougou. Patients and methods: The study was cross-sectional, from February 15th to June 30th, 2015. It was conducted in Ouagadougou (Burkina Faso). We included patients with a new central venous catheter (CVC) inserted in the hemodialysis unit. The timeout for making an arteriovenous fistula (AVF) for chronic hemodialysis in Ouagadougou may exceed six months. The CVCs used at the time of the study were made of polyurethane and non-tunneled. Sociodemographic, clinical and paraclinical data were collected and analyzed. Results: During the study period, 156 CVCs were installed in 114 patients, and the average age was 41.8 \pm 17.1 years. A CRI occurred on 39 CVCs for a rate of 9.5/1000 days-catheter. The frequency of CRI was $28.4 \%, 20.6 \%$ and $20 \%$ respectively in femoral, jugular, and subclavian veins. The culture of the tip of the catheter was done in 21 cases. Fifteen germs have been identified: eight cases of Gram-positive cocci (53.3\%) and seven cases of Gram-negative bacilli (46.7\%). It was essentially staphylococci (eight cases). In univariate analysis, a period of use of the CVC in chronic hemodialysis over 10 days was significantly $(p=0.01 ; r=2.91)$ associated with the catheter-related infection (CRI). Conclusion: The impact of the CRI was very high in our series. The associated factor in the univariate analysis was the long period of use of the CVC. The most diligent production of AVFs at the YO-UHC would contribute to a significant reduction of the CRI.
\end{abstract}

${ }^{*}$ Corresponding author.

How to cite this paper: Coulibaly, G., et al. (2015) Factors Associated with Infection of the Central Venous Catheter for Hemodialysis in Ouagadougou (Burkina Faso). Open Journal of Blood Diseases, 5, 59-65.

http://dx.doi.org/10.4236/ojbd.2015.54009 
Keywords

\section{Burkina Faso, Catheter, Hemodialysis Delivery Systems, Infections, Staphylococcus}

\section{Introduction}

The use of central venous catheters (CVCs) of hemodialysis may be beset by various complications from which the most common is the infection. The incidence of CVC-related bloodstream infections varies from 1 to 2/1000 days-catheter and that of positive cultures of CVC is on average 7/1000 days-catheter [1]. In African countries, as in Morocco and Mali, the prevalence of infectious complications related to the use of CVCs of hemodialysis varies from 39\% to 50\% [2] [3]. The Infectious complications are associated with a risk of deaths estimated to be between 4 and $20 \%$ [4].

In the unit of hemodialysis (UH) of the department of nephrology and hemodialysis at Yalgado Ouedraogo University Hospital Center (YO-UHC), we found a particularly high frequency of catheter-related infections (CRIs) by the high number of hospitalizations they caused. Therefore, we conducted this study to evaluate the frequency of CRIs in hemodialysis patients and determine the factors associated in a context of very limited resources.

\section{Patients and Methods}

The study was cross-sectional and descriptive, from February 15 to June 30, 2015. It was conducted in the unique UH, located in YO-UHC Ouagadougou (Burkina Faso). Patients taken from this hemodialysis unit were involved in this study. We have included all patients who had a new CVC inserted in the unit during the study period. The cases of change of CVCs on guide were not included.

At the time of the study, the UH had 29 hemodialysis stations for about 250 patients. The activities of the unit were carried out by 21 dialysis nurses, one care unit supervisor, and five physicians (three nephrologists and two generalists). The latter additionally provided medical activities in the clinical nephrology unit with a capacity of 24 beds.

The vascular access used in the UH were the arteriovenous fistulas (AVFs) in case of chronic hemodialysis, or CVCs for acute hemodialysis or pending creation of an AVF. The AVFs are created by the surgeons of YO-UHC. For material issues in YO-UHC and sometimes financial reasons related patients, the timeout of the AVF could reach six months or more.

The CVCs used at the time of the study were short-lived, made of polyurethane and tuneless. Their length was 15 or $20 \mathrm{~cm}$. The CVC measuring $15 \mathrm{~cm}$ were preferentially inserted into the internal jugular or right subclavian veins; those measuring20 $\mathrm{cm}$ were inserted into the internal jugular, left subclavian or femoral veins. The tunneled CVC were not yet available neither used in the unity. The unit did not have ultrasound.

The CVCs were inserted by doctors of the service in a room dedicated to this activity, but also to the dressing of the newly created hemodialysis AVFs. These dressings were not always clean. The operator as well as the aid was equipped with sterile gloves and an orinasal mask. After a surgical handwashing, he proceeded to their friction with a water-alcohol gel disinfectant before wearing sterile gloves; the aid used the same procedure in the event of need. The antiseptic solution used for skin disinfection of the patient was the povidone iodine. The dressing of the CVC was done with sterile compresses and a non-sterile adhesive dressing.

The rehabilitation of the dressing of the CVC was done during the session of acute hemodialysis or chronic (every four days) by the nurses of the UH. It was done outside of a hemodialysis session in case of soiling, purulence or detachment of the dressing. The CVC was withdrawn after use or with the occurrence of complications. The culture of the tip of the CVC was performed if the financial possibilities of the patient allowed us to do so. It should be noted that tests and drug costs, for the moment, were fully under the patient's charge.

Concerning the CVC-related infection (CRI), we selected the following operational definitions:

- The infection of the insertion hole: inflammation limited to the outlet of the CVC with suppuration. The culture of the tip of the CVC if possible, confirms the diagnosis;

- The suspicion of CVC-related bacteremia: presence of general septic signs (fever, chills, profuse sweating, tachycardia, and hypotension) associated with clear local signs like the suppuration of the orifice of the in- 
sertion of the CVC site without another source of apparent infection. Symptoms regress after antibiotic treatment or ablation of the CVC. The culture of the tip of the CVC if possible, confirms the diagnosis.

The incidence of the CRI has been expressed in "1000 days-catheters", calculated according to the following formula:

[Number of new cases of CRI of the period/ Total number of days of central venous cannulation of the period] $\times 1000$

Sociodemographic, clinical and paraclinical data were recorded and analyzed using Epi Info 7.1.3.3. The quantitative variables were expressed by their average \pm standard deviation. The ANOVA test was used for comparison of quantitative variables; for the comparison of qualitative variables or semi quantitative, $\mathrm{X}^{2}$ unadjusted test has been used for the theoretical effectives $\geq 5$ and Fisher's exact test for the theoretical effectives $<5$. The statistical differences observed have been regarded as significant for $\mathrm{p}<0.05$.

The consent of the patients (or their representative if the patient was unconscious) was obtained before their inclusion. They have been informed of the objectives and constraints of the study, necessary monitoring measures. The study data were collected in the strict respect of anonymity and confidentiality.

\section{Results}

\subsection{General Data}

During the study period, 156 CVCs were installed in 114 patients (68 men and 46 women). The average age of the patients was $41.8 \pm 17.1$ years (extreme $=5$ and 85). It was $41.9 \pm 17.1$ years for men and $41.5 \pm 17.3$ for women. The socio-economic level was low for 73 patients (64\%), average for 30 patients (26.3\%), and high for 11 patients $(9.7 \%)$.

The personal history of patients is summarized in Table 1.

\subsection{Catheter-Related Infections}

A CRI occurred with 39 CVCs representing 25\% of the 156 CVCs. That represented a rate of 9.5/1000 dayscatheter. The 39 cases of CRI were divided into:

- An infection of the outlet: four cases representing 10.3 per cent of the 39 cases of CRI and $2.6 \%$ of the 156 CVCs;

- A suspicion of CVC-related bacteremia: 35 cases representing $89.7 \%$ of the 39 cases of CRI and $22.4 \%$ of the 156 CVCs.

The CRI concerned 25CVCs inserted in a femoral vein representing 64.1 per cent, 13in an internal jugular vein (33.3\%) and 1 in a subclavian vein (2.6\%). According to the site, the CRI were more frequent at the level of the femoral vein (28.4\% of CVCs in femoral vein) compared to the internal jugular veins (20\%) or subclavian veins (20\%) (Table 2). However, this result was not statistically significant $(\mathrm{p}=0.5)$.

\subsection{Bacteriological Study}

From the 39 patients with a CRI, 21 (53.8\%) were able to culture the tip of the CVC. In 13 cases out of 21,

Table 1. Frequency of medical history of the patients.

\begin{tabular}{ccc}
\hline Personal Medical History & Number of patients & Percentage \\
\hline Hypertension & 62 & 54.4 \\
Thrombophlebitis on central venous catheter* & 13 & 28.9 \\
Diabetes & 9 & 7.9 \\
Gout & 6 & 5.3 \\
Human immunodeficiency virus infection & 3 & 2.6 \\
Lambda AL Amyloidosis & 1 & 0.9 \\
\hline
\end{tabular}

\footnotetext{
*: The research was done in 45 patients in chronic hemodialysis.
} 
Table 2. The number of infections associated with central venous catheter (CVC) according to the insertion site.

\begin{tabular}{cccc}
\hline Site of insertion & Number of CVC infected & Number of CVC inserted per site & Percentage \\
\hline Femoral vein & 25 & 88 & 28.4 \\
Internal jugular vein & 13 & 63 & 20.6 \\
Subclavian vein & 1 & 5 & 20 \\
Total & 39 & 156 & 25 \\
\hline
\end{tabular}

$\mathrm{p}=0.5$.

representing 61.9\%, at least a germ has been identified. The culture was positive in 13 patients and 15 germs have been identified. They were gram positive cocci in 8 cases (53.3\%) and in 7 cases gram-negative bacilli (46.7\%). The germs found were:

- Staphylococcus: Staphylococcus coagulase-negative staphylococci (6 cases), Staphylococcus aureus (2 cases);

- Escherichia coli: 2 cases;

- Proteus sp (2 cases) and Proteus mirabilis (1 case).

- Pseudomonas sp: 1case;

- Klebsiella oxytoca: 1 case.

\subsection{Factors Associated to CRI}

In univariate analysis, the long duration of use of the CVC was significantly associated with the presence of a CRI. The association of the CRI to the type of hemodialysis was at the limit of significance (Table 3).

\section{Discussion}

By lack of financial resources, many patients were unable to honor the request of the catheter tip culture. For the same reasons, the blood cultures were not done. The criteria of definition of the CRI which we used, allow us to diagnose patents cases; the incidence that we report is therefore probably underestimated. Nevertheless, we got interesting results which have enabled us to achieve our goals.

Compared to the AVF, the CVC infects 20 to 30 times more [5]. The incidence of infections is 0.2 per 1000 days of use for the native AVFs, 0.12 per 1000 days of use for prosthetic FAVs and 0.56 per 1000 days of use for the CVCs. According to the K-DOQI [6], the incidence of infections of CVC of hemodialysis is of the order of 2 episodes per 1000 days/patient, with extremes ranging from 1.6 to 5.5 episodes per 1000 days/patient. It varies from one country to another and from one center to another. This relatively wide range is explained in part by the multiple definitions of vascular access infections found in the recommendations. It also depends on the type of CVCs and the deep vein used. Some venous sites, such the femoral vein for example, have an increased risk of infection because of their proximity with the anal and urinary openings [7].

The incidence of 9.5/1000 days-catheter of CRI in our series was particularly high. Indeed, the incidence usually found in hemodialysis centers is the order of 2 episodes for 1000 days-patients, with extremes from 1.6 to 5.5 explained by the variability in the definition of CRI [2] [6] [8]. Our results are largely explained by the poor conditions of insertion and management of CVCs in our context of inadequate human resources, equipment and specific sterile consumables.

The ultrasound guidance is not yet possible in the UH because the ultrasound is not available. Its absence has as a consequence frequent failures of venipuncture and therefore many septic handlings. In addition, the climate in the city of Ouagadougou is quite often hot and dry, causing frequent sweating of patients, and therefore at a higher risk of contamination of the dressing that is not waterproof. Finally, the dressing of CVCs is usually done the days of hemodialysis, meaning every four days in the case of chronic hemodialysis. The used antiseptic is the povidone iodine which is certainly less costly than chlorhexidine but with inferior efficacy in preventing colonization of the CVC's inlet port or bacteremic infection [9]-[11].

The risk of infection is less for the tunneled CVCs compared to the non-tunneled CVCs [12]. The tunneled CVCs could be a good alternative in the case of chronic hemodialysis if the dressings could be frequently done. 
Table 3. Factors associated to the catheter-related infection in univariate analysis.

\begin{tabular}{|c|c|c|c|}
\hline & Presence of CRI & Absence of CRI & $\mathbf{P}$ \\
\hline \multicolumn{4}{|l|}{ Gender } \\
\hline Male & $24(27.0)$ & $65(73.0)$ & \multirow[t]{2}{*}{0.3} \\
\hline Female & $15(22.4)$ & $52(77.6)$ & \\
\hline \multicolumn{4}{|l|}{ Age } \\
\hline$\leq 20$ years $(\mathrm{n}=17)$ & $3(17.7)$ & $14(82.3)$ & \multirow{4}{*}{0.3} \\
\hline$] 20-40]$ years $(n=63)$ & $20(31.8)$ & $43(68.2)$ & \\
\hline ]40 - 60] years $(n=55)$ & $13(23.6)$ & $42(76.4)$ & \\
\hline$>60$ years $(n=21)$ & $3(14.3)$ & $18(85.7)$ & \\
\hline \multicolumn{4}{|l|}{ Socioeconomic status } \\
\hline High $(\mathrm{n}=12)$ & $4(33.3)$ & $8(66.7)$ & \multirow{3}{*}{0.3} \\
\hline Average $(n=44)$ & $14(31.8)$ & $30(68.2)$ & \\
\hline Low $(n=100)$ & $21(21.0)$ & $79(79.0)$ & \\
\hline \multicolumn{4}{|l|}{ Diabetes } \\
\hline yes $(n=13)$ & $1(7.7)$ & $12(92.3)$ & \multirow[t]{2}{*}{0.3} \\
\hline No $(n=146)$ & $22(15.1)$ & $124(84.9)$ & \\
\hline Average duration of use (days) & $42.6 \pm 35.4$ & $25.8 \pm 27.5$ & 0.004 \\
\hline \multicolumn{4}{|l|}{ Type of hemodialysis } \\
\hline Chronic $(\mathrm{n}=132)$ & $36(27.3)$ & $96(72.7)$ & \multirow[t]{2}{*}{0.06} \\
\hline Acute $(n=24)$ & $3(12.5)$ & $21(87.5)$ & \\
\hline \multicolumn{4}{|l|}{ Duration of use in $\mathrm{CH}$} \\
\hline Mean \pm SD (days) & $45.1 \pm 35.8$ & $30.5 \pm 29.3$ & \multirow{3}{*}{$\begin{array}{c}0.02 \\
0.01^{*}\end{array}$} \\
\hline$\leq 10$ days $(\mathrm{n}=23)$ & $3(13.0)$ & $20(87.0)$ & \\
\hline$>10$ days $(n=87)$ & $33(37.9)$ & $54(62,1)$ & \\
\hline \multicolumn{4}{|l|}{ Duration of use in AH (days) } \\
\hline Mean \pm SD (days) & $13.0 \pm 7.0$ & $9.2 \pm 8.1$ & \multirow{3}{*}{$\begin{array}{l}0.4 \\
0.5\end{array}$} \\
\hline$\leq 10$ days $(\mathrm{n}=16)$ & $2(12.5)$ & $14(87.5)$ & \\
\hline$>10$ days $(n=8)$ & $1(12.5)$ & $7(87.5)$ & \\
\hline
\end{tabular}

AH: acute hemodialysis; CH: chronic hemodialysis. CRI: catheter-related infection. CVC: central venous catheter. *: $r=2.91$.

Our study found no significant difference in the CRI between the CVC insertion sites. The same observation was made during randomized or nonrandomized studies. These studies have not reported an increase in the risk of bacteremia associated with femoral catheterization compared to the jugular catheterization [13]-[15]. However, the femoral CVC has the disadvantage of most often promoting deep thrombophlebitis of the lower limb [16].

Study of factors in univariate analysis has revealed only the usage duration of CVC. Thus, the risk is high beyond 10 days of use as in the study of Doukkali in Morocco [2]. The duration of use is very long in our series, especially in case of chronic hemodialysis. This is largely related to the long timeout for the production of AVF of hemodialysis. The CVC is therefore used as long as a complication was not detected. The fastest production of AVFs would contribute to a reduction of the prevalence of CVCs and the CRI. In our context of long-term use of non-tunneled CVC, the use of "locks" containing antibiotics like gentamicin associated with citrate [17] or $60 \%$ ethanol associated with heparin could also contribute to reducing the incidence of CRIs.

The most commonly encountered germs in our study were primarily Gram-positive cocci (61.5\%). This family of bacteria is indeed the most frequently reported in CRIs; and the authors agree with us that it is a staphylococcus in the majority of cases [2] [18] [19]. Staphylococcus aureus is the most frequently identified germ, in more than 70\% of cases according to some authors [15] [20] [21]. However, our results are consistent with other authors who have noticed a predominance of Staphylococcus non-aureus [22] [23].

In some cases, the nature of the isolated microorganism can lead to the source of the infection. The majority of CVCs' contamination from skin flora or fitting is due to coagulase-negative staphylococci. On the other hand, the isolation of a Staphylococcus aureus or enterobacteria orients rather towards a colonization of the equipment from a septic focus [24]. The frequency of coagulase-negative staphylococcus in our series is probably due to a lack of personal body hygiene.

In our series, we have a non-negligible frequency of gram negative bacteria (46.7\%). These germs have for 
natural habitat the nostrils (Pseudomonas) or the digestive tract (Escherichia coli, Klebsiella, Proteus). During the hemodialysis sessions, oronasal masks are not available for patients; also the hot climate associated with the dust does not often promote good body hygiene. This set of factors favors the multiplication of germs on the skin and as such would increase the risk of contamination of CVCs. The use of these masks and a better body hygiene should contribute to the reduction of contamination of these CVCs by germs that are present in these environments.

\section{Conclusion}

The impact of the CRI was particularly high in our series. It did not depend on the insertion site. The associated factor that we found in the univariate analysis was the long period of use of the CVC. The main identified germ was Staphylococcus. The most diligent production of AFVs at the YO-UHC, the availability in sufficient quantity of sterile equipment and the improvement of the working conditions in the hemodialysis unit associated with continuous awareness of patients for better personal hygiene would contribute to a lessening of the CRI. Ulterior studies could help us to determine the place of silicone or tunneled catheters and in the choice of antiseptic solution in reducing the incidence of the ILC in our context.

\section{Acknowledgements}

We thank Dr. Manan Hien for translating the text into English.

\section{Conflict of interest}

None.

\section{References}

[1] (2007) 12th Conference of Consensus in Resuscitation and Emergency Updating 2002 Medicine. Definitions of the IAS CTIN-ILS.

[2] Jon, B. (2012) Central Venous Catheters Temporary Hemodialysis. Doctoral Thesis in Medicine, Faculty of Medicine and Pharmacy, Sidi Mohammed Ben Abdellah University, Morocco. Resuscitation 2012, No. 125112.

[3] Ould Bezeïd, M.M. Assessment of Activities of the Hemodialysis Unit of National Hospital of Point "G” from 1999 to 2001. Medicine, Mali 2003, Thesis. http://www.keneya.net/fmpos/theses/2003/med/pdf/03M05.pdf

[4] Ge, X., Cavallazzi, R., Li, C., Pan, S.M., Wang, Y.W. and Wang, F.L. (2012) Central Venous Access Sites for the Prevention of Venous Thrombosis, Stenosis and Infection. Cochrane Database of Systematic Reviews 2012, Issue 3.

[5] Uzan, M. and Avignon, A. (2012) The Central Venous Catheter for Hemodialysis: Recommendations Connections/Disconnections. 8th Annual Day of Exchanges of Network DIALIN Hospital Center South Lyon, Pierre Bénite, 18 September 2012.

[6] K-DOQI (2006) Clinical Practice Guidelines for Vascular Access. American Journal of Kidney Diseases, 48, 5274-5276.

[7] Oliver, M.J., Callery, S.M., Thorpe, L.E., Schwab, S.J. and Churchill, D.N. (2000) Risk of Bacteremia from Temporary Hemodialysis Catheters by Site of Insertion and Duration of Use: A Prospective Study. Kidney International, 58, 2543-2545. http://dx.doi.org/10.1046/j.1523-1755.2000.00439.x

[8] Leou, S.F., Tarela Garnier, P.C., Ramana Lumbroso, S., Shoemaker, C., et al. (2013) French Evaluation of Infectious Complications Associated with Central Venous Catheters for Hemodialysis in Polynesia. Néphrologie \& Thérapeutique, 9, 137-142. http://dx.doi.org/10.1016/j.nephro.2013.01.003

[9] Humar, A., Ostromecki, A. and Direnfeld, J. (2000) Prospective Randomized Trial of 10\% Povidone-Iodine versus $0.5 \%$ Tincture of Chlorhexidine as Cutaneous Antisepsis for Prevention of Central Venous Catheter Infection. Clinical Infectious Diseases, 31, 1001-1007. http://dx.doi.org/10.1086/318145

[10] Paglialonga, F., Consolo, S., Biasuzzi, A., Assomou, J., Gattarello, E., Patricelli, M.G., et al. (2014) Reduction in Catheter-Related Infections after Switching from Povidone-Iodine to Chlorhexidine for the Exit-Site Care of Tunneled Central Venous Catheters in Children on Hemodialysis. Hemodialysis International, 18, S13-S18. http://dx.doi.org/10.1111/hdi.12218

[11] Mimoz, O., Lucet, J.C., Kerforne, T., Pascal, J., Souweine, B., Goudet, V., et al. (2015) Skin Antisepsis with Chlorhexidine-Alcohol versus Povidone Iodine-Alcohol, with and without Skin Scrubbing, for Prevention of Intravascular-Catheter-Related Infection (CLEAN): An Open-Label, Multicentre, Randomised, Controlled, Two-by-Two Fac- 
torial Trial. The Lancet, 386, 2069-2077. http://dx.doi.org/10.1016/S0140-6736(15)00244-5

[12] Maki, D.G., Kluger, D.M. and Crnich, C.J. (2006) The Risk of Bloodstream Infection in Adults with Different Intravascular Devices: A Systematic Review of 200 Published Prospective Studies. Mayo Clinic Proceedings, 81, 1159-1171. http://dx.doi.org/10.4065/81.9.1159

[13] Parienti, J.J., du Cheyron, D. and Timsit, J.F. (2012) Metaanalysis of Subclavian Insertion and Non-Tunneled Central Venous Catheter Associated Infection Risk Reduction in Critically Ill Adults. Critical Care Medicine, 40, 1627-1634.

[14] Marik, P.E., Flemmer, M. and Harrison, W. (2012) The Risk of Catheter Related Bloodstream Infection with Femoral Venous Catheters as Compared to Subclavian and Internal Jugular Venous Catheters: A Systematic Review of the Literature and Metaanalysis. Critical Care Medicine, 40, 2479-2485. http://dx.doi.org/10.1097/CCM.0b013e31823e99cb

[15] Dugué, A.E., Levesque, S.P. and Fischer, M.O. (2012) Vascular Access Sites for Acute Renal Replacement in Intensive Care Units. Clinical Journal of the American Society of Nephrology, 7, 70-77. http://dx.doi.org/10.2215/CJN.06570711

[16] McGee, D. and Gould, M. (2003) Preventing Complications of Central Venous Catheterization. The New England Journal of Medicine, 348, 1123-1133. http://dx.doi.org/10.1056/NEJMra011883

[17] Cote, D., Lok, C.E., Battistella, M. and Vercaigne, L. (2010) Stability of Trisodium Citrate and Gentamicin Solution for Catheter Locks after Storage in Plastic Syringes at Room Temperature. Canadian Journal of Hospital Pharmacy, 63, 304-311. http://dx.doi.org/10.4212/cjhp.v63i4.934

[18] Fram, D., Okuno, M.F.P., Taminato, M., Ponzio, V., Manfredi, S.R., Grothe, C., et al. (2015) Risk Factors for Bloodstream Infection in Patients at a Brazilian Hemodialysis Center: A Case-Control Study. BMC Infectious Diseases, 15, 158. http://dx.doi.org/10.1186/s12879-015-0907-y

[19] Katneni, R. and Hedayati, S.S. (2007) Central Venous Catheter-Related Bacteremia in Chronic Hemodialysis Patients: Epidemiology and Evidence-Based Management. Nature Clinical Practice Nephrology, 3, 256-266.

[20] Amor, S., Zellama, D., Belkhir, W., Guedri, Y., Mrabet, S., Sabri, F., et al. (2014) Epidemiological Study of Bacterial Infections Related to Jugular Catheters in Hemodialysis. Néphrologie \& Thérapeutique, 10, 294. http://dx.doi.org/10.1016/j.nephro.2014.07.009

[21] Hoen, B., Paul-Dauphin, A., Hestin, D. and Kessler, M. (1998) EPIBACDIAL: A Multicenter Prospective Study of Risk Factors for Bacteremia in Chronic Hemodialysis Patients. Journal of the American Society of Nephrology, 9, 869-876.

[22] Khosroshahi, H.T., Mahdipur, H., Parkhideh, S., Basmenji, S., Khalilzadeh, M. and Tozihi, M. (2015) The Effectiveness of Systemic Antibiotic Therapy with and without Ethanol-Locked Solution in the Treatment of Hemodialysis-Related Catheter Infection. Saudi Journal of Kidney Diseases and Transplantation, 26, 477-481. http://dx.doi.org/10.4103/1319-2442.157315

[23] Misanovic, V., Jonuzi, F., Anic, D., Halimic, M. and Rahmanovic, M. (2015) Central Venous Catheter as Vascular Approach for Hemodialysis-Our Experiences. Materia Socio Medica, 27, 112-113. http://dx.doi.org/10.5455/msm.2015.27.112-113

[24] Nitenberg, G., Jagot, J.L. and Antoun, S. (1991) Pathophysiology and Epidemiology of Infections Associated with Central Venous Catheters. Nutrition \& Metabolism, 5, 11-24. 